\title{
Neural Networks based solution for Door Automation
}

\author{
Monalika Padma Reddy \\ $8^{\text {th }}$ semester, UG Student \\ Department of Computer Science and Engineering \\ Visvesvaraya Technological University \\ monalika.6671@gmail.com
}

\begin{abstract}
Face Recognition is one of the most common biometric strategies which has gained popularity because of the accuracy and security. This paper presents the implementation of a Convolution Neural Network architecture for door automation. This model is devised to overcome the disadvantages of a traditional door system and other methods such as door automation using Bluetooth, figure prints, passwords, or retinal scans. It allows the authorized people to gain access to the house by face recognition. The proposed system makes use of convolution neural network architectures and RaspberryPi. The ResNet architecture [6] is used to implement face recognition and runs on RaspberryPi. The images of the residents of the house will be used to train the model. If the person is a resident of the house, the face will be recognized and the lock will open, else it will be recognized as a human and an alarm will ring and an email alert consisting of the image of the person in front of the door will be sent to the owner. It has numerous advantages as it is user-friendly especially for senior citizens, lesser maintenance, does not require the residents to carry the keys and reduces the threat of robbery.
\end{abstract}

Keywords: Smart Door Automation, Convolution Neural Network, face recognition, ResNet architecture

\section{INTRODUCTION}

In the traditional door systems that are being used in our residences, the number of problems being encountered is more.

Usually, it is essential for a person who is at home to leave all his work and come down to the main door to open it. The person in some cases may not be to come down to open the door due to illness or such scenarios. To overcome such cases this smart door system is being designed.

The techniques used in the implementation of the Smart Door System are:

\section{$\checkmark$ Convolution Neural Networks \\ $\checkmark \quad$ Sensor and alarm technology.}

The Convolutional Neural Network is a type of neural network that uses a mathematical operation called convolutions which is a specialized kind of linear operation. They can be referred to as neural networks that adopt convolution in place of the general matrix multiplication in at least one of their layers.

A convolutional neural network consists of three layers- namely the input layer, the hidden layer, and the output layer. There can be multiple hidden layers in the convolutional neural network that normally consist of a series of convolutional layers. The activation function that is commonly used here is RELU which is then followed by pooling layers, fully connected layers, and normalization layers which are the additional convolutions. The inputs and the outputs of these layers are masked by the activation function and final convolution and hence, these are referred to as the 
hidden layers. It must be noted that the hidden layers in a convolutional neural network must be fully connected.

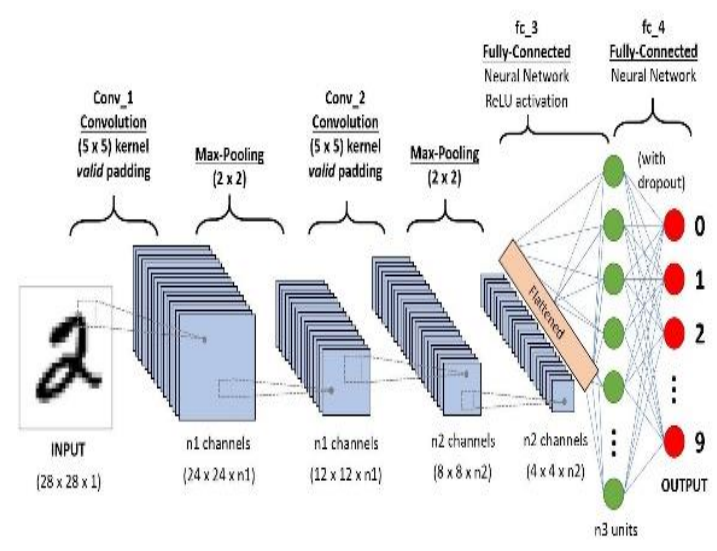

Fig: 1 Convolution Neural Network

A sensor is a device the typically responds to a stimulus and transmits a resulting impulse for operating a control. The stimulus to a sensor can be in the form of heat, magnetism, or a particular motion. The image sensors are the most popular combination of OpenCV vision software and webcam that can be used for face detection and facial recognition. An impulse will be generated by the sensor which will either open the door or sound an alarm.

Tensorflow Lite is a set of tools that are used to run TensorFlow models on IoT, embedded, and mobile devices. It enables on-device machine learning inference with a small binary size and low latency.

\section{LITERATURE REVIEW}

\section{A. Password Based Security Lock System}

In this model [1], the door automation is done using passwords. The person who wants to gain access to the house must enter a 4 digit password. If the password matches, then the person will be granted access. However, this method is prone to hacking, the human tendency of forgetting the password, and mechanical failures. If the 4 digit password is hacked, then security will be compromised. In case the owner forgets the passwords, then there will be a huge problem.

\section{B. Bluetooth Smart Radar Door Systems Via Mobile Apps}

In this model [2], Bluetooth is used as a wireless connection protocol for the automation of the door lock system. The drawback of this system is it does not provide any emergency keys and this is feasible only if the person has a smartphone. In case the person does not have a smartphone or if the battery is dead or the smartphone is lost, then this model will not work.

\section{Remote Monitoring Intelligent System Based on Fingerprint Door Lock}

In this model, [3] fingerprint is usually for enforcing security. Here, the model identifies the lively fingerprint accurately and the unlock information ID, illegal burglary information to the owner over a GSM network. This method of using fingerprints for door locks has disadvantages such as software malfunctions, scanner issues, fake positives, and other major security breaches.

\section{IRIS Biometric Recognition for Person Identification and Security}

In this model, the identification of a person is usually done using the iris scan [4]. The hamming distance is used for calculating the distance between the input images and the images in the database and then two different classifiers are used for the identification of a person before granting access. However, it is difficult to obtain the small target from a distance. Iris tends to deform non elastically with the change in the pupil size and the illumination must be bright or visible.

\section{NEURAL NETWORKS}

\section{A. The Neuron}

Neurons, popularly known as nerve cells send and receive signals from the brain. These are structurally and functionally unique that the other cells in the body. An axon is a specialized projection that allows the neurons to transmit electrical and chemical signals to other cells. 
The dendrites are root-like structures that branch out from the head of the neuron that process and receive signals from other neurons. In other words, dendrites are receivers and axons are transmitters for signals.

\section{Structure of a Typical Neuron}

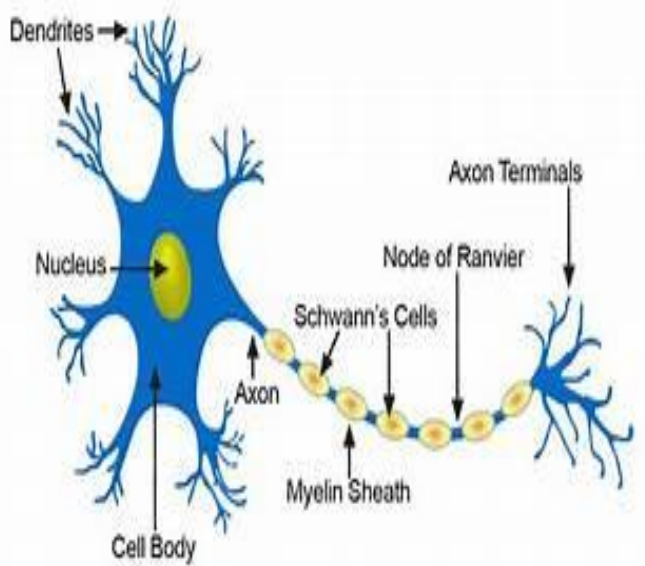

\section{Fig:2 Structure Of a Neuron in the human brain}

\section{B. Representation of neurons in Artificial Intelligence}

A neuron can technically be termed as a node that receives an input signal and produces a corresponding output signal. The input signals can be obtained from other neurons as well and these are called input layers.

The input signal is the independent variable of the model, such as the age of a person. It is essential for standardization and normalization to be done on the input variables.

The obtained output values can be continuous (like the price value) or binary( $\mathrm{Y}$ or $\mathrm{N}$ like a person purchasing a house or not) or categorical. Each signal consists of weights.

The weights play a significant role because neural networks learn by adjusting the weights by themselves. The importance of a signal will be decided by the weights associated with that signal.

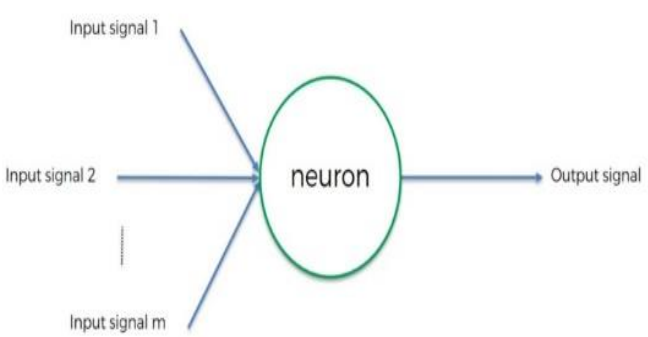

Fig: 3 Representation of Neurons

\section{Working of Neural Network in Artificial Intelligence}

There are 3 main steps that are involved in the working of a neural network.

Here, in the first step, all the weights are added and multiplied with the independent variables and a bias is added. The basic formula that is used for this is

$\mathrm{Y}=\mathrm{w} 1 * \mathrm{x} 1+\mathrm{w} 2 * \mathrm{x}+\ldots+\mathrm{wm}^{*} \mathrm{xm}+$ Bias where $\mathrm{m}$ represents the number of input layers.

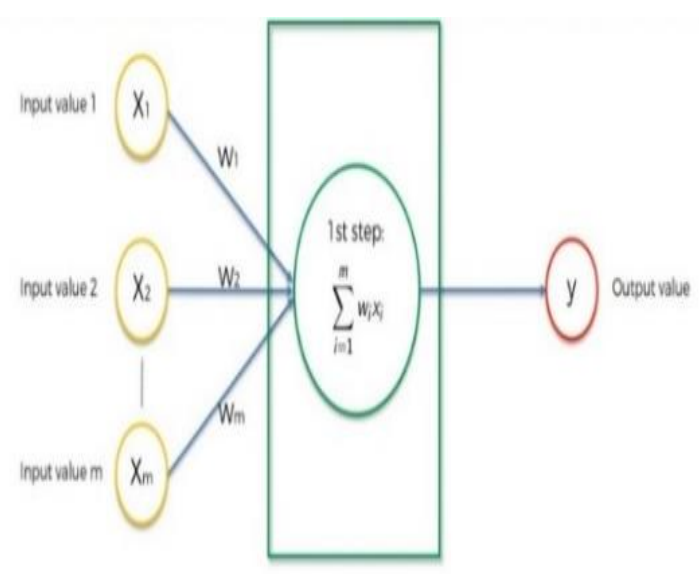

Fig: 4 Working of a Neural Network

In the second step, the activation function is applied. The four popularly known activation functions are threshold function, sigmoid function, rectified function, and hyperbolic function. The activation function is used to determine which signal will pass on to the output layer.

In the third step, signals are sent to the output layers. The above three steps are called forward propagation. 


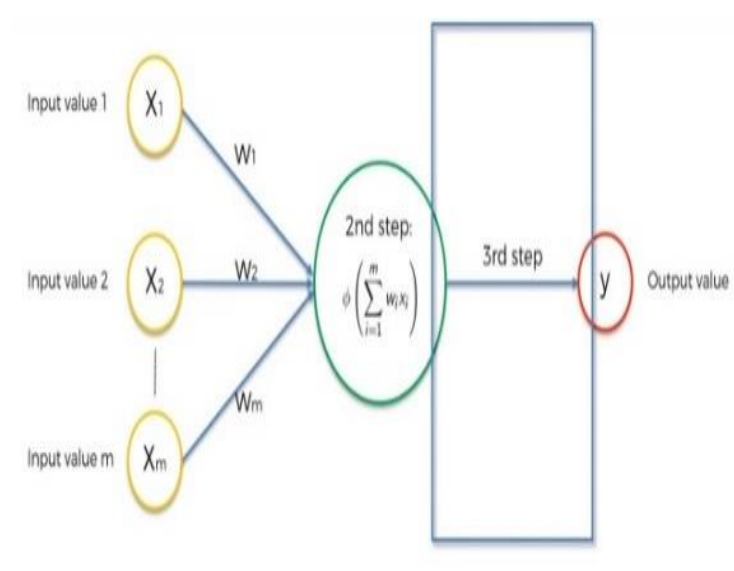

Fig: 5 Neural Network final step

The output, thus obtained is called as y predicted which is compared with the actual value of $y$, and the weights are updated using the chain rule. This is called backpropagation. The errors that are present are calculated using the loss function. The loss function uses the crossentropy to compute the error which is then backpropagated into the neural network for it to learn. Along with the weights, feature detectors are also adjusted. It is important to note that the data goes through the whole network, the error is calculated and backpropagated.

\section{FACE DETECTION USING CONVOLUTION NEURAL NETWORKS}

There is a staggering degree of similarity between the working of the human brain and the working of a convolution neural network.

\section{A. Cerebral cortex and visual cortex}

The human brain is divided into four parts out of which one is the cerebral cortex. It is a thin layer that covers the outer portion of the brain. The visual cortex is present inside the cerebral cortex. The visual cortex is a part of the cerebral cortex that receives and processes the impulses from the optic nerves.

\section{B. Similarities between the convolution neural network and human brain}

Suppose we are seeing a dog, then that information that is in the form of an impulse is passed from the sensory organ - eyes into the brain with the help of optic nerves. This will then be further passed into several neurons and finally to the visual cortex in the cerebral cortex region. Let us assume that the visual cortex has several layers say $\mathrm{v}_{1}, \mathrm{v}_{2}, \mathrm{v}_{3}$, and so on. These layers play a vital role. Suppose the $v_{1}$ layer is responsible for identifying the edge of the dog like the body edge, it calculates it.

Then, the obtained information is passed on to the next layer $\mathrm{v}_{2}$ where information of the movements of the dog or if any other object is present beside it and is responsible for distinguishing the dog and the other object. On similar lines, the information is gathered by the different subsequent layers and passed on. It can be observed that the different operations that are being carried out here are called filters in convolution neural network

\section{CONVOLUTION NEURAL NETWORKS}

The convolution neural network has two important parts- namely, feature extraction and classification. A series of convolution and pooling operations will be performed by the network in feature extraction during which the features will be detected.

The fully connected layer in the convolution neural network will serve as a classifier. The probability for the object on the image that is being predicted by the algorithm will be assigned. There are four important steps that are involved in face recognition. They are:

$$
\begin{array}{ll}
\checkmark & \text { Convolution } \\
\checkmark & \text { Pooling } \\
\checkmark & \text { Flattening } \\
\checkmark & \text { Full Connection }
\end{array}
$$

\section{A. Convolution}

Convolution is a function that is obtained integration of two given functions which expresses how one function is modified by the other. The mathematical formula for convolution is as given below 


$$
(f * g)(t)=\int_{-\infty}^{\infty} f(\tau) g(t-\tau) d \tau
$$

Fig: 6 Convolution function

Input image, feature detector, and feature maps are the three essential elements that enter into the convolution operation.

The basic idea of the convolution is Input image $\mathrm{x}$ Feature detector $=$ Feature Map.

The main advantage of using feature maps is reducing the size of the input image, making it easier to read. A convolution matrix to adjust an image. It can be used to blur, edge detect, and sharpen the image.

The primary purpose of convolution is to find features in an image using feature detectors, put them into a feature map, and having them in the feature map. The spatial relationship between the pixels is preserved. The images on the computer are represented in 0's and 1's.

Consider the image having a $6 \times 6$ matrix and a filter having a $3 \times 3$ matrix. As discussed earlier, layer $\mathrm{v} 1$ was responsible for detecting the edge of the image.

On similar lines, the vertical edge filter is represented by the filter i.e. the vertical edge of the image can be determined by applying this filter on that particular image.

Multiply the first $3 \times 3$ matrix with filter and add. The same procedure should be repeated by moving by one. This is an iterative process and once the end is reached, it should be stepped down by one, and the same procedure must be continued.

A $4 \times 4$ matrix is obtained as the result of the matrix multiplication. This operation is called Convolution.

\begin{tabular}{|l|l|l|l|l|l|}
\hline 0 & 0 & 0 & 1 & 1 & 1 \\
\hline 0 & 0 & 0 & 1 & 1 & 1 \\
\hline 0 & 0 & 0 & 1 & 1 & 1 \\
\hline 0 & 0 & 0 & 1 & 1 & 1 \\
\hline 0 & 0 & 0 & 1 & 1 & 1 \\
\hline 0 & 0 & 0 & 1 & 1 & 1 \\
\hline
\end{tabular}$*$\begin{tabular}{|l|l|l|}
\hline 1 & 0 & -1 \\
\hline 2 & 0 & -2 \\
\hline 1 & 0 & -1 \\
\hline
\end{tabular}

Image Filter

\begin{tabular}{|l|l|l|l|}
\hline 0 & -4 & -4 & 0 \\
\hline 0 & -4 & -4 & 0 \\
\hline 0 & -4 & -4 & 0 \\
\hline 0 & -4 & -4 & 0 \\
\hline
\end{tabular}

Result

The feature detector is also known as the filter or a kernel. These play a vital role. Just like the vertical filter, we have many filters that can be used for detecting the edge of the face, horizontal edge filter, and so on.

From the resultant matrix, it can be concluded that the image size $n=6$ and filter size $\mathrm{f}=3$. The formula for the result can be devised as follows.

\section{Result $=\mathbf{n}-\mathbf{f}+\mathbf{1}$}

Hence, the result for image size $n=6$ and filter size $\mathrm{f}=3$ is $6-3+1=4$

From the result, a $4 \times 4$ matrix is obtained but the size of the image is a $6 \times 6$ matrix. It can be seen that chances some of the vital information exists. To avoid this, the concept of padding is applied.

\section{B. Padding}

The two main downsides of convolution are the shrinking of outputs and the loss of information, especially in the corners of the image. To overcome this, padding is done.

In simple words, it can be described as an additional layer that can be added to the border of the image. This helps in increasing the accuracy.

$n-f+1=6$

$n=6-1+f$ 


$$
\mathbf{n}=\mathbf{5}+\mathbf{3}=\mathbf{8}
$$

The value of $n$ obtained here is 8 . If a padding $\mathrm{p}=1$ is applied then, we get an image of size $8 \times 8$ is obtained. The following is the result obtained on multiplying the image with the filter.

$$
\text { Result }=\mathbf{n}+\mathbf{2 p}-\mathbf{f}+\mathbf{1}
$$

Result $=6+2-3+1=6$

The resultant matrix, thus obtained is $6 \times 6$ and there is no loss of data. The original size of the image can be retained if padding is applied. Any number of convolution and padding can be applied to retain the size of the image.

\section{Pooling}

The neural networks have a property called spatial invariance which means that the location of a feature in an image doe not matter. The main objective of pooling is to reduce dimensionality, down-sample an input representation, and allowing the assumptions made about the features contained in the sub-regions. It can be described as a sample-based discretization process. Here, the max pooling feature is applied. The maximum value of the pixels is taken and placed over a separate matrix. Pooling is of many types, namely sum pooling, average pooling, and subsampling. Subsampling is the generalization of mean pooling. Max pooling is a type of pooling where only the high pixel values are being taken and hence, we can recognize the face in the image.

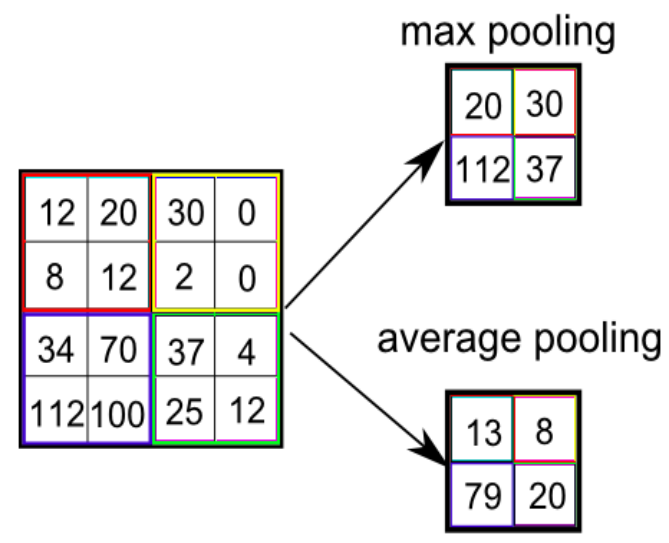

Fig: 7 Types of Pooling

\section{Flattening}

Flattening can be defined as the process of converting all the resultant twodimensional arrays into a single long continuous linear vector.

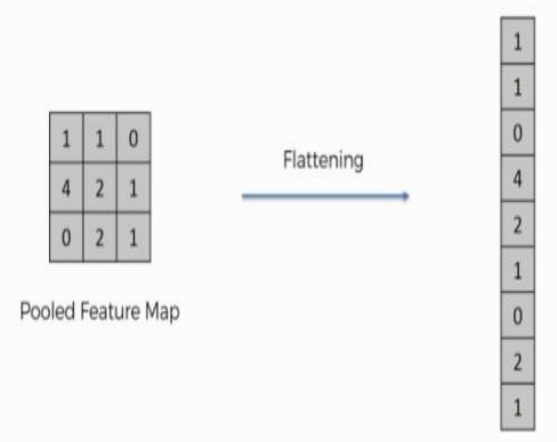

Fig: 8 Flattening

\section{E. Fully-Connected layer}

A way of learning non-linear combinations of features of a higher level as represented by the output of the convolution layer can be done by adding a fully connected layer.

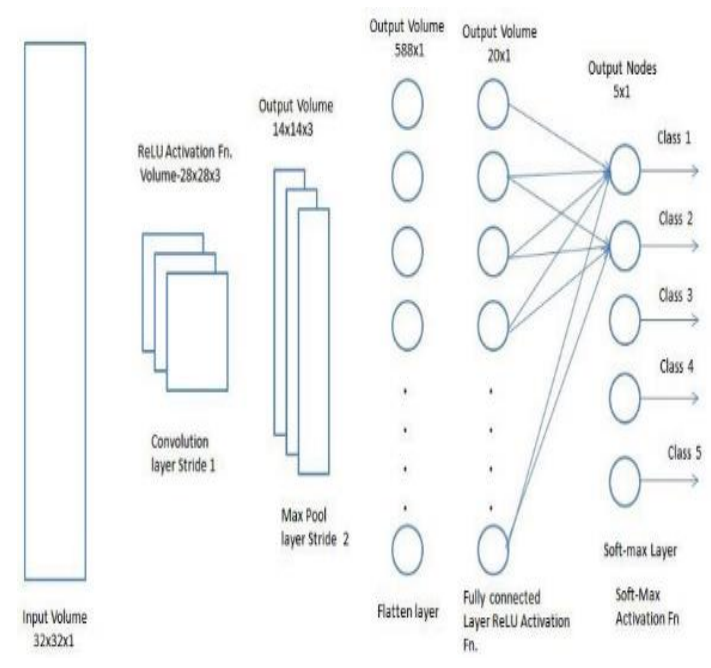

Fig: 9 A fully connected layer

\section{CNN ARCHITECTURES}

Convolution Neural Networks are a class of neural networks used in analyzing visual imagery. They can be used for image classification, object detection, segmentation, and other image processing tasks.

Many CNN architectures were developed and can be used to improve the accuracy of predictions in custom $\mathrm{CNN}$ models. 
The CNN architectures are as follows:

$\checkmark$ VGG Net

$\checkmark$ ResNet

$\checkmark$ Dense Net

$\checkmark$ Inception Net

$\checkmark$ Xception Net

\section{A. VGG Net [5]}

It uses $3 \times 3$ convolution layers that are stacked one above the other in increasing depth. Max Pooling is used to reduce the volume size. It consists of two fully connected layers and uses a softmax classifier. VGG16 and VGG19 are VGG nets where 16 and 19 refer to the number of weighted layers in the network and are consisted very deep. It is used mainly used in deep learning image classifications. However, VGG Net is very slow to train and the weights of the network architecture are large. VGG Net is also prone to the vanishing gradient problem.

\section{B. ResNet [6]}

It is short for Residual Networks and used quite often in computer vision tasks. The use of skip connections is to mitigate the vanishing gradient problem by providing an alternate shortcut for the gradient and adding output from the previous layers to the later layers. ResNets have many variants. However, the basic idea is to consider the output of a Conv2D layer and add the output to the Conv2D layer and then send this as an input to the next layers. The stacked layer is added along with its input layer. The training is usually done using the batch normalization layers, which will facilitate the model to train faster by boosting the weights and minimize the vanishing gradient problem. The size of the model is smaller because the global average pooling is used rather than fully connected layers, which reduces the model size dramatically.

\section{Dense Net [7]}

The connectivity pattern is simplified in Dense Nets. They require a fewer number of parameters and the layers are quite narrow and add a small ser of feature maps. They do not add the output feature maps of a layer but concatenate them with the incoming feature maps. They usually have more intermediate connections. The vanishing gradient problem is minimized as well. Smaller filter counts can be used and are good for smaller models.

\section{Inception Net [8]}

The main goal of Inception Net is to behave as a multi-level feature extractor. It computes $1 \times 1,3 \times 3$, and $5 \times 5$ convolutions in the network module and the output of these are stacked along the dimension of the channel before being fed into the subsequent layers. Inception Nets are preferred because they are wider and many such layers can be stacked and the output parameters to be trained are much less.

\section{E. Xception Net [9]}

Xception Net stands for extreme inception. It is basically an improvisation of the Inception Net in terms of computational efficiency. The main difference between the two is that in Inception Net normal convolutional operations are performed but in Xception Net, depth-wise separable convolutions are performed. By using depth-wise separable convolutions, the computational complexity can be reduced because each kernel is two-dimensional and they are convoluted over a single channel only. Xception Nets can be used in lowpower devices and have fewer computations in the Conv Layer.

\section{FACE DETECTION}

Face detection is a computer technology that has wide areas of applications in everyday life. They are solely used for the identification of human faces in digital images. They can specifically be regarded as a case of object class detection. Face detection can be done using neural networks which is part of artificial intelligence, where a machine can learn by itself without explicitly being programmed by a programmer.

The concept of face detection is used in biometrics and often as a part of the facial recognition system. Here, it is essential to train the machine with the images of the 
residents so that machine will learn to detect the faces of residents by itself.

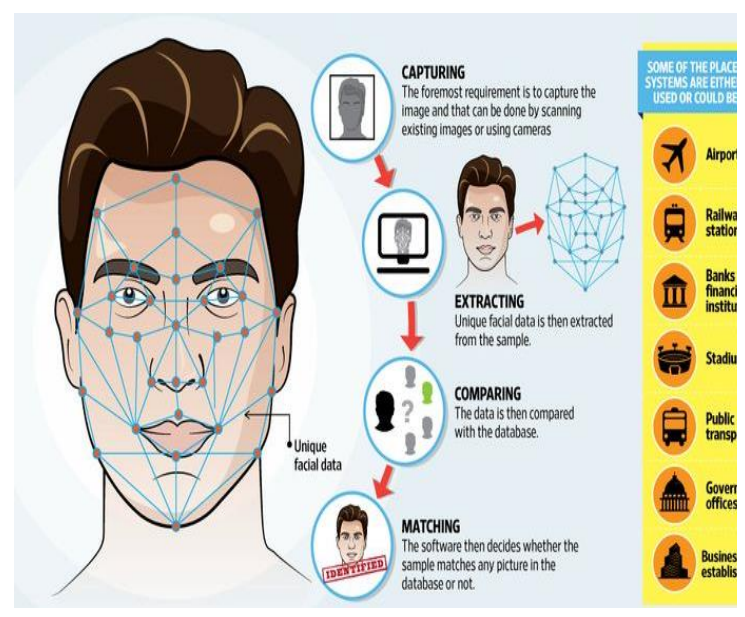

Fig: 10 Face Detection

\section{PROPOSED MODEL}

Locks have played an important role in the security of residential and industrial sectors. Especially at houses, they have acted as a secure foundation for centuries. The locks are usually taken for granted and eventually they have faded into the background of security, even though the significance held by them is still great. Many people don't realize how important locks are, until they are either locked out of their own houses, or they find themselves unable to lock their residential doors. It is also a tedious job especially for the senior citizens to get up and open the door. The chances of theft are also relatively high in the case of these traditional door lock systems. To overcome the disadvantages of fingerprint door locks, Bluetooth door locks, face recognition can be used as a mechanism of door unlock.

In this system, images of the people residing in the house will be fed and it will be trained using a ResNet architecture. If the person is a resident of the house, the face recognition will be performed and the door will unlock and the person is let in. In case the person is not a member residing in that house but comes to visit the house, the person's face will be recognized as a human and an alarm will ring indicating that some human being who is not a member of that house is at the door and an email alert will be sent to the owner consisting of the image of the person in front of the door.

\section{METHODOLOGY}

Tensor flow (Keras) is used for image recognition. The techniques that are being implemented are:

1. Collection of images

2. Image pre-processing and image normalization

3. Building $\mathrm{CNN}$ architectures

4. Training the model

5. Saving the inference models in both Tensorflow and Tensorflow Lite

6. Integrating the model saved in Tensorflow Lite with RaspberryPi

7. Face recognition

8. Unlocking the door or generating an alarm and sending an email notification to the owner.

\section{OBSERVATIONS}

When a person is detected, the model will recognize the human being by using face detection. If it is a resident the door will automatically unlock. Otherwise, an alarm will ring indicating that the person is an outsider and an email notification with the person's image will be sent to the owner. It can thus be used to prevent any kind of drawbacks that arise in the case of traditional door lock systems.

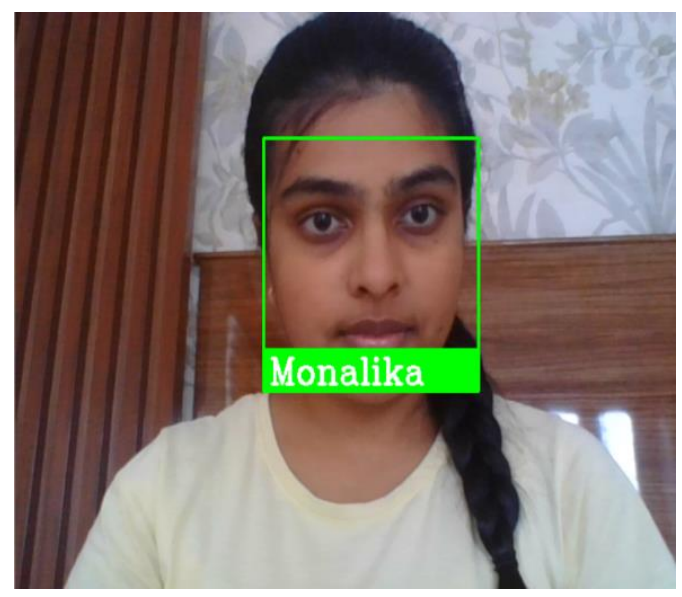

Fig: 11 Face detection and recognition

Fig 11 shows the implementation of the face recognition. On integration with 
Raspberry Pi, the door will be unlocked for the authorized user. In case, the person is not a recognised user, the alarm will ring and an email notification will be sent to the owner.

\section{CONCLUSION}

The proposed model can be used to provide a safe and secure residential home there, solving all the drawbacks of the traditional door lock systems. By using the ResNet architecture for face recognition, it improves the accuracy to a great extent. The size of the model is also less and hence, it is easier to integrate it with RaspberryPi. The requirement to train the system is also quite less and hence is optimal and feasible. It also enables a full automation system with minimal human effort or interaction, such as opening the door and it eliminates a majority of the security threats. Door automation using face recognition is also no contact and is easy to use even for age groups. It also makes it easier to track down thieves and trespassers because if the person is not recognized as a resident, then the email alert with the person's image will be sent to the owner.

\section{REFERENCES}

[1] Arpita Mishra, Siddharth Sharma, Sachin Dubey, S.K. Dubey, "Password Based Security Lock System", International Journal of Advanced Technology in Engineering and Science, 2011.

[2] Muhammad Yusry Bin Ishak, Samsiah Binti Ahmad, Zalikha Zulkifli, “ Iot Based Bluetooth Smart Radar Door Systems Via Mobile Apps", 1st International Conference on Artificial Intelligence and Data Sciences (AiDAS), 2019

[3] Wu Ping, Wu Guichu, Xie Wenbin, Lu Jianguo, Li Peng, "Remote Monitoring Intelligent System Based on Fingerprint Door Lock", International Conference on Intelligent Computation Technology and Automation, 2010

[4] S.Vinitha, R. Karthiyan, "IRIS Biometric Recognition for Person Identification and Security", International
Journal of Scientific Research in Computer Science, Engineering and Information Technology, Volume 2, 2017

[5] Karen Simonyan, Andrew Zisserman, "Very Deep Convolutional Networks for Large Scale Image Recognition" Conference paper, ICLR, 2015

[6] Kaiming He, Xiangyu Zhang, Shaoqing Ren, Jian Sun, "Deep Residual Learning for Image Recognition", Proceedings of the IEEE Conference on Computer Vision and Pattern Recognition (CVPR), 2016, pp. 770-778

[7] Gao Huang, Zhuang Liu, Laurens van der Maaten, Kilian Q. Weinberger, "Densely Connected Convolutional Networks", Proceedings of the IEEE Conference on Computer Vision and Pattern Recognition (CVPR), 2017, pp. 4700-4708

[8] Christian Szegedy, Wei Liu, Yangqing Jia, Pierre Sermanet, Scott Reed, Dragomir Anguelov, Dumitru Erhan, Vincent Vanhoucke, Andrew Rabinovich, "Going Deeper With Convolutions", Proceedings of the IEEE Conference on Computer Vision and Pattern Recognition (CVPR), 2015, pp. 1-9

[9] François Chollet, "Xception: Deep Learning with Depthwise Separable Convolutions", IEEE Conference on Computer Vision and Pattern Recognition (CVPR), 2017

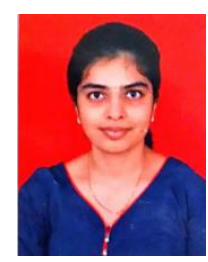

Monalika Padma Reddy undergraduate student pursuing a Bachelor's degree in Computer Science and Engineering (2021 pass out) at the Visvesvaraya Technological University, Belagavi. Her research interests include machine learning, deep learning, computer vision and natural language processing. 